\title{
Captive chimpanzee foraging in a social setting: a test of problem solving, flexibility, and spatial discounting
}

Lydia M Hopper, Laura M Kurtycz, Stephen R Ross, Kristin E Bonnie

In the wild, primates are selective over the routes that they take when foraging and seek out preferred or ephemeral food. Given this, we tested how a group of captive chimpanzees weighed the relative benefits and costs of foraging for food in their environment when a less-preferred food could be obtained with less effort than a morepreferred food. In this study, a social group of six zoo-housed chimpanzees (Pan troglodytes) could collect PVC tokens and exchange them with researchers for food rewards at one of two locations. Food preference tests had revealed that, for these chimpanzees, grapes were a highly-preferred food while carrot pieces were a lesspreferred food. The chimpanzees were tested in three phases, each comprised of 30, 30minute sessions. In phases 1 and 3 , if the chimpanzees exchanged a token at the location they collected them they received a carrot piece (no travel) or they could travel $\geq 10 \mathrm{~m}$ to exchange tokens for grapes at a second location. In phase 2, the chimpanzees had to travel for both rewards ( $\geq 10 \mathrm{~m}$ for carrot pieces, $\geq 15 \mathrm{~m}$ for grapes). The chimpanzees learned how to exchange tokens for food rewards, but there was individual variation in the time it took for them to make their first exchange and to discover the different exchange locations. Once all the chimpanzees were proficient at exchanging tokens, they exchanged more tokens for grapes (phase 3). However, when travel was required for both rewards (phase 2), the chimpanzees were less likely to work for either reward. Aside from the alpha male, all chimpanzees exchanged tokens for both reward types, demonstrating their ability to explore the available options. Contrary to our predictions, low-ranked individuals made more exchanges than high-ranked individuals, most likely because, in this protocol, chimpanzees could not monopolize the tokens or access to exchange locations. Although the chimpanzees showed a preference for exchanging tokens for their more-preferred food, they appeared to develop strategies to reduce the cost associated with obtaining the grapes, including scrounging rewards and tokens from group mates and carrying more than one token when travelling to the farther exchange location. By testing the chimpanzees in their social group we were able to tease apart the social and individual influences on their decision making and the interplay with the physical demands of the task, which revealed that the chimpanzees were willing to travel farther for better. 
2 Lydia M Hopper ${ }^{1,2, *}$, Laura M Kurtycz ${ }^{1}$, Stephen R Ross ${ }^{1}$, \& Kristin E Bonnie ${ }^{1,3}$

3

4 1. Lester E. Fisher Center for the Study and Conservation of Apes, Lincoln Park Zoo, Chicago, IL, USA

5 2. Department of Psychology, Northwestern University, Chicago, IL, USA

6 3. Department of Psychology, Beloit College, Beloit, WI, USA

7

8 *Corresponding author: Lydia M. Hopper (Ihopper@lpzoo.org) 
10 How, when, where, and for how long animals forage for food is influenced by external factors (e.g., predation

11 risk, social interactions, and prey availability); internal factors (e.g., hunger, food preferences, and animals' age

12 or sex); and phylogenetic factors (e.g., physiological parameters and sensory limitations) (Pianka, 1997). The optimal foraging theory proposes that foraging should increase fitness while reducing foraging costs (Pyke, 1984; Bautista, Tinbergen \& Kacelnik, 2001) and suggests that animals should prefer to travel a shorter, rather than a longer, distance to obtain food if all other options are equal (Blaser \& Ginchansky, 2012; Reilly et al., 2012). However, if the food that is farther away is 'better' (e.g., larger, more plentiful, more preferred) than the closer option, will animals exert more effort to obtain the better food? Humans, for example, will travel farther to reach their more-preferred restaurants (Froehlich et al., 2006), and we show such behavioral choices in other contexts too, including travelling farther to visit more closely related kin (Pollet, Roberts \& Dunbar, 2013) and to reach hospitals that offer higher quality healthcare (Romley \& Goldman, 2011). Like humans, nonhuman primates not only have individual preferences for certain foods, but seek them out within their environment (Janmaat, Byrne \& Zuberbühler 2006; Janmaat, Ban \& Boesch, 2013), suggesting that they would be likely to travel farther for better.

In the natural habitat of primates, food sources are not evenly distributed, either physically or temporally. In order to obtain preferred or ephemeral foods, primates are selective about which trees they feed from (Glander, 1978) and the routes that they travel to reach them (Ban, Boesch \& Janmaat, 2014). Primates also appear willing to travel farther to obtain their more-preferred foods, even if less-preferred foods are closer or easier to obtain. For example, observations of wild black howler monkeys (Alouatta pigra) suggests that their foraging strategy is influenced by both distance (they preferentially travel to closer trees) and food desirability (they use step-wise movements to reach high quality patches, Plante, Colchero \& Calmé, 2014). Similarly, wild capuchins (Cebus apella nigritus) have been shown to weigh the relative cost of distance and food availability such that they will detour from a direct path to food only when it enables them to gain 
33 additional, and worthwhile, food items (Janson, 2007). Female chimpanzees (Pan troglodytes) have been

34 reported to leave their nighttime nests earlier when more-preferred fig trees are located farther away

35 (Janmaat et al., 2014), indicating that they are willing to travel longer distances to reach more desirable food

36 rewards and that they plan their activities in order to reach them. Even in a captive setting, where primates are

37 not required to forage for their daily food to survive, they have been reported to use efficient routes when

38 searching for foods, sometimes bypassing less-preferred foods to reach more-preferred foods first (e.g.,

39 Menzel, 1973, Boesch \& Boesch, 1984 provides a review), but evidence for such strategic foraging is mixed

40 (e.g., Howard \& Fragaszy, 2014).

Many primates live in social groups and so an individual's foraging strategy and food choices may also

be influenced by the decisions and preferences of its group mates as well as the effort required to obtain that food (Finestone et al., 2014; Hardus et al., 2015; Marshall et al., 2015). Indeed, an advantage of social living for gregarious primate species is that they can use social information to learn where to find food (Rappaport \& Brown, 2008), which foods to eat (Visalberghi \& Addessi, 2000; van de Waal, Borgeaud \& Whiten, 2013), and how to process those foods (Boinski \& Timm, 1985; van de Waal, Bshary \& Whiten, 2014). In experimental tests, chimpanzees have been shown to copy the foraging techniques of their group mates (e.g., Whiten, Horner \& de Waal, 2005), even when the technique appears arbitrary (Bonnie et al., 2007). Furthermore, tests with socially-housed captive chimpanzees have identified that low-ranking and 'uncertain' chimpanzees appear more likely than dominants to use social information (Kendal et al., 2015), and they preferentially copy dominant or 'expert' individuals (Horner et al., 2010), even when doing so means they received a lesspreferred food reward (Hopper et al., 2011). Therefore, although tests with individual primates can reveal how they weigh the physical costs of foraging (e.g., Stevens et al., 2005), studies run with socially-housed captive primates can also uncover how they weigh the social costs of foraging along with the physical costs. Previous tests of socially-housed captive primates' foraging have assessed their ability to learn novel extractive foraging tasks from observing others (e.g., P. troglodytes, Whiten, Horner \& de Waal, 2005; Saimiri 
57 boliviensis, Hopper et al., 2013), but only a few have offered differentially-valued food rewards in such research paradigms (e.g., Dean et al., 2012). Two such studies (Hopper et al., 2011; van Leeuwen et al., 2013) presented chimpanzees with opportunities to exchange tokens for food rewards in a group setting, a technique most notably utilized in tests of social learning (e.g., Bonnie et al., 2007; Horner et al., 2010). In these two experiments, chimpanzees were given the option to exchange tokens for two differently-valued foods, revealing that they could discriminate between the two options (Hopper et al., 2011; van Leeuwen et al., 2013). Unlike in the examples of wild primate foraging described above, in these two studies the effort required to obtain both rewards was the same, even though they were valued differently by the chimpanzees. In Hopper and colleagues' (2011) study, chimpanzees could exchange different colored tokens that were assigned different reward values. As the chimpanzees collected both token types at the same location and exchanged them all with a researcher at a second location 8m away, the effort required was equal irrespective of which color token the chimpanzees exchanged and, therefore, which reward they received (less-preferred carrot piece or more-preferred grape). Similarly, in van Leeuwen and colleagues' (2013) study, chimpanzees could collect tokens from within their enclosure and then trade them at one of two locations, one where they received a high-value reward (5 peanuts) or one where they received a low-value reward (1 peanut). However, as the distance between the two exchange locations and the token collection point was the same (30m), the effort required to obtain either reward value was also the same. Although not designed to test the impact of the physical environment on the chimpanzees' food exchanged their tokens with a researcher at a single location, subordinate chimpanzees could not easily avoid competition and often had tokens taken from them by their group mates (especially those tokens that

79 garnered the high-value grapes). Although all the chimpanzees had shown an individual preference for one 80 food type (grapes), certain low-ranking chimpanzees often exchanged the tokens that were worth the less- 
81 preferred rewards (carrot pieces). Hopper and colleagues (2011) concluded that the chimpanzees switched 82 away from their preferred technique in order to ameliorate competition while still obtaining food rewards, 83 even if they were the less-preferred option. Flexibility of a different kind was revealed by van Leeuwen and colleagues' (2013) study. Despite having previous personal experience of exchanging tokens for food rewards

85 at a certain location, when a second exchange location became more profitable, the chimpanzees switched strategies and exchanged tokens at the new location where they could obtain more rewards for each exchange. The chimpanzees preferentially exchanged tokens where they could obtain more rewards, even though they had to switch from a previously-learned strategy, something it has been suggested that primates fail to do when both options are equal (e.g., C. apella, Brosnan \& de Waal, 2004; P. troglodytes, Hrubesch, Preuschoft \& van Schaik, 2009). Extending upon these token exchange methods and to test whether a group of captive chimpanzees would choose to travel farther to obtain a more-preferred reward, we provided a group of chimpanzees with a single location where they could collect PVC tokens and two locations where they could exchange the tokens with researchers for differentially-valued foods. Crucially, the distance between the token collection point and the two exchange locations was different, such that the chimpanzees had to travel farther to obtain their more-preferred food. As we also wanted to test whether they would be able to shift their foraging locations We had a number of key aims with this study, all of which related to how the individual chimpanzees foraged in a dynamic social and physical environment. Accordingly, we also had a number of predictions.

101 Chimpanzees can learn how to exchange tokens very quickly when given training (e.g., Hopper et al., 2011; van Leeuwen et al., 2013) or from watching group mates (Bonnie et al., 2007). Beyond this, in previous studies that have presented socially-housed primates opportunities to exchange tokens for food rewards, dominant individuals monopolized access to tokens (P. troglodytes, Bonnie et al., 2007) and made more exchanges with 
105 them (C. apella, Addessi et al., 2011).Therefore, our first prediction was that all the chimpanzees would learn

106 how to exchange the tokens with researchers for food rewards, either through individual trial-and-error

107 learning or by using social information, and that, once the chimpanzees had learned to exchange tokens for

108 food, higher-ranking chimpanzees would exchange more tokens than low-ranking individuals. Accordingly, the

109 chimpanzees received no training prior to the start of this study either in how to exchange tokens or where the

110 exchange locations were.

111 Previous research has demonstrated that primates can associate different types of tokens with

112 different associated food rewards (C. apella, Brosnan \& de Waal, 2004; Addessi, Crescimbene \& Visalberghi,

113 2007; Addessi et al., 2011; P. troglodytes, Hopper et al., 2011, van Leeuwen et al., 2013) and that chimpanzees

114 will selectively exchange tokens at specific locations in order to obtain more desirable rewards when the two

115 options are equally accessible (van Leeuwen et al., 2013). Extending this, we were interested in the likelihood

116 of the chimpanzees traveling farther for the more-preferred rewards and if they would continue to do so when

117 the locations where they could exchange tokens for food rewards changed. Our second prediction, therefore,

118 was that the chimpanzees would exchange more tokens for their more preferred foods, even though they had

119 to travel farther to do so. However, as previously comparable studies have shown that chimpanzees attempt to

120 obtain tokens or rewards opportunistically, by scrounging them from their group mates (Hopper et al., 2011;

121 van Leeuwen et al., 2013), our third prediction was that the chimpanzees would scrounge from one another

122 and that dominant individuals would be more likely than subordinates to scrounge tokens and food rewards

123 from their group mates.

125 Materials and Methods

126 Subjects and Housing

127 This study was conducted with six zoo-born, mother-reared chimpanzees (two males, four females) housed

128 together in one social group at the Lincoln Park Zoo, Chicago, USA. At the start of the 15-month study, the 
129 average age of the chimpanzees was 19.8 years (range $=13.2-28.4$ years). The chimpanzees participate in

130 regular cognitive testing in a group setting using touchscreen interfaces (Wagner \& Ross, 2013) and their tool-

131 use behavior has also been extensively studied (e.g., Lonsdorf et al., 2009; Bonnie, Ross \& Lonsdorf, 2012;

132 Calcutt et al., 2014; Hopper et al., 2015). However, at the start of this study, the chimpanzees were naïve to

133 exchanging PVC tokens with researchers in order to obtain food rewards and the two researchers who ran the

134 experimental sessions (LMH and LMK) had not previously worked with this particular group of chimpanzees.

135 Therefore, the chimpanzees had no prior experience receiving food rewards from these researchers or

136 exchanging the PVC tokens with animal care staff or researchers for food rewards.

137 The chimpanzee group was housed in an expansive indoor-outdoor enclosure at the Regenstein Center

138 for African Apes at Lincoln Park Zoo, which incorporated climbing structures, deep-mulch bedding and an off-

139 exhibit holding area. The indoor exhibit measured $408 \mathrm{~m}^{2}$ and the outdoor exhibit measured $2011 \mathrm{~m}^{2}($ total $=$

$1402419 \mathrm{~m}^{2}$ ). Throughout the study, the chimpanzees had outdoor access when weather conditions were

141 appropriate $\left(>5^{\circ} \mathrm{C}\right)$ but all testing was conducted in their indoor enclosure. In addition to any food rewards that

142 the chimpanzees obtained during the course of this study, fresh produce and primate chow were scattered

143 twice daily throughout their exhibits. Zoo guests were able to observe every test session, and trained

144 educators interpreted every test session to communicate the importance of cognitive testing with the animals.

\section{Ethics}

147 This study was approved by the Lincoln Park Zoo Research Committee, which is the governing body for all

148 animal research at the institution. No social group manipulations occurred as the result of this project. Food

149 substances, amount, and frequency were reviewed and approved by veterinary and nutrition staff prior to the

150 start of the project. No modifications were made to standard animal care routines. This research adhered to

151 legal requirements in the United States of America and to the American Society of Primatologists' Principles for

152 the Ethical Treatment of Nonhuman Primates. 


\section{Food Preference Testing}

155 Before testing began, food preference tests were run with the six chimpanzees to determine their more-

156 preferred and less-preferred foods. As chimpanzee food choices are known to be affected by social influences

157 (Finestone et al., 2014), and because we tested the chimpanzees in a group setting, we tested each

158 chimpanzee's individual food preferences while in the presence of their group mates, (c.f. Hopper et al., 2011).

159 Food preferences were determined using a forced-choice paradigm (c.f. Hopper et al., 2014a). Chimpanzees

160 were each offered two different food items presented on long wooden skewers held at shoulder width by a

161 member of animal care staff. As soon as a chimpanzee selected one of the two food items, by reaching through

162 the cage mesh with their fingers or mouth, the member of animal care staff gave them that food item and

163 withdrew the other option. Ten such tests were run on one day and ten more on a second day with each of the

164 six chimpanzees. These tests revealed that each of the six chimpanzees selected grapes over similarly-sized

165 carrot pieces $\geq 80 \%$ of the time. To determine that the chimpanzees would eat carrot pieces when no other

166 food options were available, on a third day the chimpanzees were offered only carrot pieces and all the

167 chimpanzees readily ate all the carrot pieces given to them. Thus, grapes were used throughout this study as

168 the more-preferred food rewards and carrot pieces as the less-preferred food rewards.

169

\section{Procedure}

171 Within each test session, the chimpanzees had to collect tokens $(10 \mathrm{~cm}$ long lengths of $2.5 \mathrm{~cm}$ diameter white

172 PVC pipe) from a single location. The chimpanzees could then exchange the tokens with researchers at one of

173 two locations on the perimeter of their indoor exhibit, one where they could get a piece of carrot for each

174 token exchanged (CLOSE location) and one where they could get a grape for each token exchanged (FAR

175 location, Figure 1). The distance from the location where the chimpanzees collected the tokens to the two

176 exchange locations was different such that the chimpanzees had to travel farther to reach the FAR location 
177 than to reach the CLOSE location (Figure 1 provides details of the specific distances). The entire study was

178 subdivided into three phases (1, 2, and 3). To measure the chimpanzees' behavioral flexibility, we varied the

179 location of the exchange locations across the phases (and therefore also the effort required to reach each

180 location), as shown in Table 1 and Figure 1. Each of the three phases was comprised of 30, 30-minute, test

181 sessions ( 90 sessions in total $=45$ hours), with two test sessions run per week (on Tuesday and Thursday

182 mornings from 11:30-12:00). The study lasted 15 months, and data were collected between January 2013 and

183 April 2014.

184

185

\section{FIGURE 1 AND TABLE 1 ABOUT HERE}

186

hoppers (plastic milk crates) hung side-by-side on the human side of a panel of mesh at the edge of the

chimpanzees' enclosure (location A, shown in Figure 1). The chimpanzees could easily obtain the tokens by

reaching for them through the mesh with their fingers. To ensure that the tokens could not be monopolized by

191 a single animal, the chimpanzees were provisioned with 150 tokens per session ( 25 tokens/chimpanzee) and

could collect them from either of the two hoppers. The chimpanzees were able to collect as many tokens from

193 the hoppers as they wished and exchange them with a researcher at either of the two exchange locations

194 (Figure 1). To successfully exchange a token, a chimpanzee had to completely push the token through the cage

195 mesh in front of the researcher, but they did not hand it to the researcher directly (the zoo's safety policy

196 prohibits such direct interactions between researchers and animals). In each session, a researcher stood at

197 each of the two exchange locations (CLOSE and FAR) and gave the chimpanzees a food reward for each token

198 that they exchanged. At each exchange location a clear container filled with the food rewards was fully visible

199 to any chimpanzees that approached the exchange location; at the CLOSE location the tub always contained

200 pieces of carrots and at the FAR location the tub always contained grapes. The two researchers were the only 
201 two people with whom the chimpanzees could exchange the tokens with or receive rewards from. To reduce

202 the risk of researcher bias or chimpanzee preferences for certain researchers, the researchers switched

203 locations every session. Across all phases, there was no difference in the number of tokens chimpanzees

204 exchanged with either of the two researchers at either the CLOSE or FAR location (Wilcoxon Signed Rank test:

205 CLOSE: $\mathrm{T}=-.674, \mathrm{~N}=6, \mathrm{P}=0.500 ; \mathrm{FAR}: \mathrm{T}=-0.943, \mathrm{~N}=6, \mathrm{P}=0.345)$. For each exchange that a chimpanzee

206 completed, the researcher simply gave them the appropriate food reward and did not provide any other

207 reinforcers (e.g., verbal praise) and nor did they encourage the chimpanzees to exchange tokens (e.g., by

208 holding out their hand or by calling the chimpanzees over).

\section{Coding and Analysis}

211 All sessions were filmed using Sony Handycams (HDR-CX160). At each of the two exchange locations cameras

212 on tripods were set to film the mesh panel through which the chimpanzees could exchange tokens (Figure 1).

213 This footage also captured any other chimpanzees within the vicinity of the exchange location (i.e. chimpanzee

214 'observers'). Throughout each session each of the two researchers provided a running commentary that was

215 recorded by the camera on the tripod at their location. Additionally, a third researcher with a handheld

216 camcorder stood on the public floor and filmed the chimpanzees' activities (Figure 1).

For each token that a chimpanzee exchanged, the researcher noted (1) the identity of the chimpanzee

218 who completed the exchange, (2) the food reward that they were given (grape or piece of carrot), (3) whether

219 the focal chimpanzee ate the food reward within 30 seconds, and, if not, whether another member of the

220 group ate it within 30 seconds and that chimpanzee's identity. The researchers also noted which other

221 chimpanzees, if any, observed each exchange. An 'observing' chimpanzee was defined as 'a chimpanzee that

222 was within $1 \mathrm{~m}$ of the chimpanzee making the exchange and that was also oriented towards them as they

223 completed the exchange with a researcher' (c.f. Hopper et al., 2011). The researcher also noted every time a

224 chimpanzee exchanged an item from their exhibit that was not a token (e.g., a piece of bark from the mulch 
225 floor, or a stick from a shrub in their outside enclosure) and which chimpanzees observed these exchanges, if

226 any. Exchanges of non-token items were never rewarded; the chimpanzees could only obtain food rewards

227 from the researchers by exchanging one of the 150 provided PVC tokens. Ad libitum data were also collected

228 on 'token transfers' that were observed by the researchers from the exchange locations. A token transfer was

229 defined as 'one chimpanzee taking a token directly from the possession of another chimpanzee' (sensu Hopper

230 et al., 2011) rather than collecting it from one of the two hoppers at location A or picking a token up from the

231 floor. Footage of a token transfer event can be seen here: http://youtu.be/vOWYEcYn8Wo. All data were

232 transcribed into Excel for analysis at which point the time stamp for each exchange (as gathered from the video

233 camera recording) was also logged.

234 As two researchers simultaneously collected data, after the first two sessions, both researchers coded

235 the video tape footage of both exchange locations (i.e. coding their own exchanges with the chimpanzees and

236 those made by the second researcher). The two researchers had $100 \%$ concordance in their rating of each

237 exchange that was completed and the identity of all observers. All further video footage was coded singly by

238 LMK. Additionally, we calculated the reliability of our ratings by computing intra-class correlation coefficients

239 (ICC) between the ratings completed by LMK and ratings completed by a researcher who was familiar with this

240 chimpanzee group, but who had never seen these test sessions. This researcher blind coded two randomly-

241 selected tapes of chimpanzees' exchanges at the CLOSE location and two randomly-selected tapes of

242 chimpanzees' exchanges at the FAR location for each of the three experimental phases (12 tapes in total, 360

243 minutes). There was high inter-rater reliability for all measures; ICC $(2,1)$ for the number of tokens exchanged

244 by each chimpanzee within a session at a certain location $=0.999(P<0.001)$; ICC $(2,1)$ for the number of non-

245 token items exchanged by each chimpanzee within a session at a certain location $=0.969(P<0.001)$; and ICC

$246(2,1)$ the number of observers that watched each chimpanzee's exchanges $=0.876(P<0.001)$. These inter-class

247 correlation coefficients, along with all analyses reported below were completed in IBM SPSS version 20 (IBM,

248 New York), while all graphs were produced in R (R Development Core Team, 2010) using ggplot 2 (Wickham, 
249 2009). In order to determine whether the chimpanzees' behavior (e.g., food scrounging) or their acquisition of 250 the task was related to their rank, we collected assessments of the chimpanzees' rank from four researchers

251 who work regularly with the chimpanzees (following Kendal et al., 2015). However, rather than using a

252 categorical measure of rank (i.e., high, mid, low, c.f. Kendal et al., 2015), the chimpanzees' rank was rated on a 253 linear scale from 1 (lowest ranked) to 6 (highest ranked).

255 Results

\section{Acquisition and adoption of the exchanging behavior}

257 All chimpanzees in the group exchanged multiple tokens throughout the course of the study (Figure 2). The 258 first chimpanzee to exchange a token did so during the first session of phase 1 at the CLOSE location and the 259 last chimpanzee to start participating first exchanged a token at the FAR location during the $78^{\text {th }}$ session in 260 phase 3 (locations A and B respectively, Figure 1). There was no correlation between the chimpanzees' rank 261 and the order in which they acquired this task (i.e. session in which they made their first exchange for a food 262 reward food reward: Spearmans's rho: $\left.r_{s}=-0.371, N=6, P=0.468\right)$, however, there was a negative correlation 263 between rank and number of tokens exchanged across all sessions (Spearmans's rho: $r_{s}=-1.00, N=6, P<$ 264 0.001). This pattern was reflected when considering their exchanges at the CLOSE (Spearmans's rho: $r_{s}=-$ 0.886, $N=6, P=0.019$ ) and FAR (Spearmans's rho: $r_{s}=-0.812, N=6, P=0.050$ ) exchange locations separately.

266 In addition to exchanging the PVC tokens, some chimpanzees exchanged other items from within their 267 enclosure (for which they were not rewarded). The rate of exchanging non-token items was relatively low 268 (average $=2.8$ exchanges/session, compared to an average 68.3 token exchanges/session) and the 269 chimpanzees exchanged significantly more tokens than non-token items (Wilcoxon signed ranks test: $z=-2.20$, $270 \quad N=6, P=0.028)$. 


\section{Selective exchanges for preferred rewards}

275 Beyond simply evaluating whether the chimpanzees could learn how to trade tokens for food rewards, we 276 were interested in assessing whether they showed a preference for exchanging tokens for their more-

277 preferred food reward, grapes, despite having to travel farther to obtain them. (We also analyzed two other 278 factors that might have influenced where the chimpanzees' exchanged the tokens - the number of visitors 279 present and the chimpanzees' individual food preferences - but neither of these influenced the chimpanzees' 280 behavior, see Supplemental Information 1 for these analyses.)

281 Over the 90 sessions the chimpanzees made a total of 2500 exchanges at the CLOSE locations and 3654 282 at the FAR location (see Supplemental Information 2). In phase 1, the five chimpanzees that exchanged tokens 283 exchanged more tokens at the CLOSE location for carrot pieces than at the FAR location for grapes (Wilcoxon 284 signed ranks test: $z=-2.02, N=5, P=0.043$, Table 2). In phase 2 , there was no significant difference in the 285 location where the chimpanzees exchanged tokens (Wilcoxon signed ranks test: $z=1.22, N=5, P=0.223$ ). This 286 is most likely because only three chimpanzees exchanged $>10$ tokens in this phase, but the two females that exchanged the most tokens in this phase did so at the FAR location (Table 2).I In phase 3, when the exchange locations were the same as in phase 1 (Table 1), and all six chimpanzees exchanged tokens, the chimpanzees exchanged more tokens at the FAR location for grapes than at the CLOSE location for carrot pieces (Wilcoxon signed ranks test: $\mathrm{z}=1.99, \mathrm{~N}=6, \mathrm{P}=0.046$, see Table 2 and the Supplemental Information 2).

\section{TABLE 2 ABOUT HERE}

The difference in the chimpanzees' responses between phase 1 and 3 is most likely due to the 
297 the more-preferred grapes (Figure 3; Supplemental Information 2). The chimpanzees' behavior in phase 3

298 (Figure 3) suggests that they preferred to travel $10 \mathrm{~m}$ to obtain grapes than to directly exchange tokens for

299 carrot pieces, which required no travel. We propose that their behavior in phase 3 is a more accurate reflection

300 of their choices because, by this phase, all six chimpanzees had exchanged tokens for food rewards, all had

301 greater exposure to the task, and all had discovered the FAR location. Supporting this, in phase 1 as their

302 exposure to the task increased, the number of exchanges that the chimpanzees made at the FAR location

303 increased over time. Specifically the number of tokens exchanged at the FAR location was positively correlated

304 with session number (Spearmans's rho: $r_{s}=0.858, N=30, P<0.001$ ) and conversely there was a negative

305 correlation between number of tokens exchanged at the CLOSE location and session number (Spearmans's rho:

$\left.306 r_{s}=-0.469, N=30, P=0.009\right)$.

307

308

FIGURE 3 ABOUT HERE

\section{Switching strategies and spatial discounting}

311 Aside from one male, all chimpanzees exchanged tokens at both the CLOSE and FAR locations (Figure 2). Thus,

312 even after discovering one successful method, the chimpanzees explored alternative options (Table 2). Indeed,

313 these five chimpanzees also exchanged tokens at both locations within single sessions, but there was no

314 correlation between the chimpanzees' rank and the average number of times they switched between the two

315 exchange locations within in a single session (Spearmans's rho: $r_{s}=0.200, N=5, P=0.747$ ).

316 In phase 1, three chimpanzees, who had previously received rewards for exchanging tokens at the

317 CLOSE location, went on to exchange tokens at the FAR location. None of these three chimpanzees had been

318 recorded to observe any chimpanzee exchange a token at the FAR location before they themselves first

319 exchanged a token at that location (Table 3). Furthermore, despite introducing novel exchanging locations in

320 phase 2, four chimpanzees exchanged tokens at the new FAR location (Table 2), and, aside from the first 
321 female to discover this new location, all did so after observing other chimpanzees at that location successfully

322 exchange tokens for grapes (Table 3). In phase 3, when the two exchange locations mirrored those in phase 1 ,

323 three of the chimpanzees exchanged tokens with researchers at the FAR location for the first time, having not

324 done so in phase 1, and all did so after they had observed multiple successful exchanges at this location by

325 other chimpanzees in their group (Table 3). In phase 3, five of the chimpanzees exchanged at both the CLOSE

326 and FAR locations, and continued to exchange at the CLOSE location even after discovering the FAR one (Table 327 2).

TABLE 3 ABOUT HERE

In phase 2, the reward locations were different to those in phases 1 and 3 , and in this phase the

chimpanzees were required to travel to obtain both reward types (Figure 1). The chimpanzees varied in their willingness to travel $15 \mathrm{~m}$, compared to $10 \mathrm{~m}$, to obtain the more-preferred grapes and $10 \mathrm{~m}$, compared to $0 \mathrm{~m}$, to get carrot pieces. Considering their exchanges for grapes, most of the chimpanzees made more exchanges at the FAR location in phase 3 compared to phase 1 (Table 2), most likely because they were more familiar with the task, as discussed above. In phase 2, when the effort to obtain grapes was increased, the majority of chimpanzees made fewer exchanges at the FAR location (Table 2), compared to in phases 1 and 3. One 13-year old female $(\mathrm{CH})$, however, exchanged more tokens in phase 2 at the FAR location (when she had to travel 15m) than in either phase 1 or 3 (when she only had to travel $10 \mathrm{~m}$ to obtain grapes).

\section{Strategies for reducing the effort required to obtain rewards}

342 During the study, the chimpanzees adopted three key strategies that potentially reduced the effort required to 343 obtain the rewards. One was to take tokens from other chimpanzees who had already retrieved them from the 344 token hopper and carried them to an exchange location (a 'token transfer'); a second was to eat the food 
345

346 $365=0.080)$.

reward after an exchange had been completed by another individual ( $a$ 'food scrounging' event); and a third was to carry more than one token from location A to the exchange locations. Across all three phases, there were 172 token transfer events, and although the chimpanzees exchanged the token they obtained in $84.4 \%$ of these cases, these events represented only $2.4 \%$ of the 6154 total exchanges made by chimpanzees throughout the three phases. The rate of reward scrounging was also very low: 317 food rewards were scrounged in total during the 90 sessions.

Considering the chimpanzees' scrounging behavior, there was no difference in the number of tokens chimpanzees took from their group mates across the three phases (Friedman's test: $X^{2}(2)=3.36, P=0.186$ ). In phase 3, however, chimpanzees were more likely to take tokens from their group mates and exchange them within 30 seconds for rewards at the FAR location compared to at the CLOSE location (Wilcoxon signed ranks test: $z=2.03, N=6, P=0.042)$. There was no difference, however, in the number of tokens that chimpanzees took from their group mates at the two exchange locations in either phase 1 (Wilcoxon signed ranks test: $z=-$ $0.82, N=5, P=0.414$ ) or phase 2 (Wilcoxon signed ranks test: $z=1.00, N=3, P=0.317$ ). Reflecting their token transfer behavior, there was no difference in the number of food rewards that the chimpanzees scrounged from their group mates across the three phases (Friedman's test: $X^{2}(2)=2.33, P=0.311$ ). In phase 1 , when the chimpanzees made more exchanges for carrots than grapes, the chimpanzees scrounged more carrots from their group mates at the CLOSE location than grapes at the FAR location (Wilcoxon signed ranks test: $z=-1.00$, $\mathrm{N}=6, \mathrm{P}=0.046)$. In phases 2 and 3 , when the chimpanzees exchanged more tokens for grapes, although not significant, there was a trend for the chimpanzees to scrounge more grapes at the FAR location than for carrots at the CLOSE location (Wilcoxon signed ranks test: phase $2, \mathrm{z}=1.83, \mathrm{~N}=6, \mathrm{P}=0.068$; phase $3, \mathrm{z}=1.75, \mathrm{~N}=6, \mathrm{P}$

There was no correlation between the chimpanzees' rank and the number of tokens (Spearmans's rho: $r_{s}=-0.086, N=6, P=0.872$ ) or food rewards (Spearmans's rho: $r_{s}=0.429, N=6, P=0.397$ ) that they scrounged. However, considering the two types of scrounging behavior collectively (food scrounging plus token 
369 transfers), there was a positive correlation between the chimpanzees' rank and the proportion of scrounging

370 events that were food scrounging events (Spearman's rho: $r_{s}=0.899, N=6, P=0.015$ ). Specifically, higher

371 ranked individuals were more likely to scrounge food rewards while lower ranked individuals were more likely

372 to take tokens from their group mates.

373 In phases 1 and 3, the upshot of the chimpanzees sometimes carrying more than one token with them

374 from location A to location B was that the average latency between each exchange a chimpanzee made at the

375 FAR location was not significantly different than the latency between exchanges made at the CLOSE location,

376 even though travel between exchanges was not required at the CLOSE location (Wilcoxon signed rank test:

377 phase $1, z=0.00, N=3, P=1.00 ;$ phase $3, z=1.75, N=5, P=0.08)$. In phase 2 , only one chimpanzee exchanged

378 tokens at both the CLOSE and FAR locations.

379

\section{Discussion}

381 The purpose of this study was to document the acquisition of a novel foraging paradigm involving token

382 exchange by a group of zoo-housed chimpanzees. Beyond this, we were interested in the chimpanzees'

383 flexibility to discover and exploit novel exchange locations within a social environment, and also to document

384 how effort impacted their foraging choices. As predicted, all six chimpanzees learned how to exchange tokens

385 in order to obtain food rewards from researchers, but even within this small sample of six chimpanzees there

386 was individual variation among the chimpanzees in the time it took for them to do so and their overall level of

387 participation in the study. The chimpanzees exchanged significantly more tokens than non-token items (e.g.,

388 bark chips and twigs), which suggests that they learned the contingency of exchanging tokens specifically,

389 rather than just general exchanging behavior. Once all the chimpanzees were proficient at exchanging tokens,

390 they chose to travel farther to exchange tokens for a preferred food (grapes), but sometimes adopted

391 strategies to reduce the effort per exchange, by scrounging tokens and food rewards from others and by

392 carrying more than one token at a time. However, when the distances to reach both rewards were increased in 
phase 2 (sessions 31-60), the chimpanzees were less likely to travel to obtain either reward. Finally, aside from

394 the alpha male, in phase 3 (sessions 61-90) all the chimpanzees exchanged tokens at both the CLOSE and FAR

395 locations demonstrating their ability to explore the available options, even after they had previously learned

396 one solution. It was this flexibility that allowed the chimpanzees to discover their preferred exchange location

397 where they could obtain grapes (the FAR location).

398 The chimpanzees learned the exchanging paradigm very quickly. Five of the six chimpanzees made

399 their first exchange within the first seven sessions and the first chimpanzee did so within the first four minutes

400 of the first session. These five chimpanzees all completed their first exchange at the CLOSE location, which was

401 also where they collected the tokens. Though it is possible that these individuals had previously learned

402 exchange contingencies from interactions with animal care staff, it is important to note that they were not

403 specifically trained to do so, nor did they have any experience receiving food rewards from the researchers

404 prior to this study. Intriguingly, despite the relatively quick acquisition of the required exchanging behavior by

405 the chimpanzees, the dominant male did not make his first exchange until the $78^{\text {th }}$ session. His first exchange

406 was made after he had been exposed to the experimental paradigm for 13 months and had seen his group

407 mates rewarded for 181 exchanges (including observations of exchanges at both locations in each of the three

408 phases), which suggests that his lack of participation cannot be explained by a lack of opportunities to observe

409 other individuals performing the task. It is notable that this same male was also the last to start using tools in

410 an earlier study that investigated the groups' acquisition of tool use when presented with a novel artificial

411 termite mound in a social setting (Lonsdorf et al., 2009; Hopper et al., 2015).

In the third phase, when all six chimpanzees participated in the study, they showed a preference for

413 exchanging tokens for their more-preferred rewards (grapes), even though they could obtain carrot pieces at

414 the same location where they collected the tokens. The behavior of the chimpanzees both reveals their

415 foraging preferences but also highlights their flexibility; five of the six chimpanzees exchanged at both the

416 CLOSE and FAR locations, often within single sessions. Previous studies have suggested that chimpanzees may 
417 be conservative and unable to adopt new strategies if they already know one that is rewarding (Hrubesch et

418 al., 2009), a factor that may be related to chimpanzees' lack of cumulative culture (Dean et al., 2012). However,

419 a more recent study revealed that chimpanzees were able switch away from a previously-learned location for

420 exchanging tokens, when a new one garnered more rewards (van Leeuwen et al., 2013). Our results reflect

421 this: five of the six chimpanzees exchanged at the FAR location for grapes, even though it required farther

422 travel to reach and they had previously been rewarded for exchanging tokens at the CLOSE location.

423 Like tests of temporal discounting, in which individuals are asked to choose between a small reward

424 now or a larger reward later (Evans et al., 2012), tests of spatial discounting assess whether individuals prefer

425 to travel farther to obtain a more desirable reward, and how far they are willing to travel for that reward

426 (Stevens et al., 2005; Lihoreau, Chitkka \& Raine, 2011; Perrings \& Hannon, 2011; Kralik \& Sampson, 2012). For

427 example, when given the choice to walk less far to a smaller reward or farther for a bigger reward, tamarins

428 (Saguinus oedipus) consistently selected the larger reward regardless of distance, however marmosets

429 (Callithrix jacchus) were less likely to choose the larger reward over the smaller one as the distance between

430 the two options increased (Stevens et al., 2005). The responses of the chimpanzees in phases 2 and 3 suggest

431 that they were willing to travel farther for better, but only up to a point, but other factors may also have

432 influenced their behavior. For example, while we controlled the distance that the chimpanzees had to walk, we

433 could not control for the time it took for different individuals to walk from location $A$ to $B$ (as in tests of

434 temporal discounting) and nor did we control the number of tokens that they carried per journey. Indeed, the

435 chimpanzees sometimes carried multiple tokens which had the end result of making chimpanzees' inter-

436 exchange interval at the CLOSE and FAR locations not significantly different. Thus, the chimpanzees preferred

437 to exchange tokens for grapes and developed strategies to reduce the effort required to obtain them, again

438 highlighting their flexible problem solving skills.

439 Despite having never been in close range (within $1 \mathrm{~m}$ ) of another chimpanzee exchanging tokens at the

440 FAR location, and having successfully exchanged multiple tokens themselves for pieces of carrot at the CLOSE 
441 location, three chimpanzees explored and found the FAR location in phase 1 and exchanged tokens there. Due

442 to the design of our study, we cannot know how each of these three chimpanzees discovered this novel

443 exchanging location, whether by individual trial-and-error learning or via social means. Although it is likely that

444 all three chimpanzees independently discovered this new location, especially as the test environment was their

445 familiar exhibit, as soon as the first chimpanzee exchanged a token at the FAR location, we cannot rule out the

446 possibility that the other chimpanzees to exchange there were influenced by the behavior of the first. Even if

447 these chimpanzees were never recorded as being within $1 \mathrm{~m}$ of the first female when she exchanged a token at

448 the FAR location before they themselves did, simply seeing her carrying tokens to the FAR location, in

449 combination with their personally-acquired knowledge that tokens could be exchanged with researchers to

450 obtain foods, might have been sufficient for them to learn this new option. This 'low-level' social learning

451 mechanism (i.e. local enhancement) has also been shown to influence the learning and decision-making of a

452 number of species when foraging (e.g., Mikolasch, Kotrschal \& Schloegl, 2012; Takahashi, Masuda \& Yamashita,

453 2013; Avarguès-Weber \& Chittka, 2014; Webster \& Laland, 2013) and thus is a parsimonious explanation for

454 the chimpanzees' learning here.

455

Interestingly, the first female to exchange a token at the FAR location in phase 1 was the lowest-ranked

member of the group and also the first to discover the novel FAR location in phase 2 . It is possible that this 13-

457 year old female's low rank or age may explain her exploratory behavior, as has been reported for wild

458 chimpanzees (Reader \& Laland, 2001). Rather than exhibiting behavioral inhibition in the presence of

459 dominants and ending participation altogether, as reported previously for primates (e.g., Macaca mulatta,

460 Drea \& Wallen, 1999; S. boliviensis, Hopper et al., 2013., P. troglodytes, Cronin et al., 2014), this female

461 continued to exchange tokens, but at alternative locations. Beyond this specific female, and contrary to our

462 predictions based on the findings of previous research (e.g., Bonnie et al., 2007; Addessi et al., 2011; Hopper et

463 al., 2011), in this group of six chimpanzees there was a negative correlation between rank and total number of

464 exchanges made (i.e. low-ranked individuals made more exchanges than high-ranked individuals). 
Even though the chimpanzees scrounged food and tokens from each other, the rate of scrounging that

466 we observed was considerably lower than that reported for other token exchange studies run with groups of

467 chimpanzees (e.g., Hopper et al., 2011). Furthermore, we found no correlation between rank and the total

468 number of tokens and food rewards scrounged. However, we did find that dominants were more likely to take

469 food rewards than tokens. We propose that the low rate of scrounging in our study, and the negative

470 correlation between rank and total tokens exchanged, is because, unlike the paradigm used by Hopper and

471 colleagues (2011), the chimpanzees had two spatially distant locations within their enclosure where they could

472 exchange tokens for food items. Additionally, we provisioned our chimpanzees with a greater number of

473 tokens to exchange compared to previous studies, which also likely reduced within-group competition and

474 therefore scrounging rates (e.g., this study provided $=25$ tokens/subject while, for example, Bonnie et al. 2007

475 provided 1.7 tokens/subject). Our experimental design, in combination with the small number of chimpanzees

476 that this group is comprised of, may help to explain why, unlike in previous studies (e.g., Bonnie et al., 2007;

477 Addessi et al., 2011), lower-ranking chimpanzees were able to exchange more tokens than higher-ranking

478 individuals (they could avoid dominants by exchanging at alternative locations) and levels of scrounging were

479 relatively low (tokens were not easily monopolized).

Ultimately, this study demonstrated that this group of six chimpanzees showed flexible foraging

481 strategies and the ability to explore their environment in order to find their most desired rewards. Following

482 previous tests of foraging and decision making with captive animals (e.g., Stevens et al., 2005; Lihoreau,

483 Chitkka \& Raine, 2011; Reilly et al., 2012), beyond simply testing their ability to discover reward locations, we

484 also assessed whether the chimpanzees would still attempt to obtain their more-preferred rewards when more

485 effort (i.e. the distance required to reach them) was required. The chimpanzees, to a limit, were willing to

486 travel farther to obtain the more-preferred grapes but future studies should test whether chimpanzees would

487 be willing to exert more effort for more-preferred rewards if the type of effort was different, for example time

488 to process the food or increased competition to negotiate. Unlike in previous studies with chimpanzees (e.g., 
489 Hrubesch et al., 2009; Hopper et al., 2011), the chimpanzees were not conservative, but showed the ability to

490 transition between different solutions in order to maximize their rewards (see also Manrique, Völter \& Call,

491 2013; van Leeuwen et al., 2013; Yamamoto, Humle \& Tanaka, 2013).

492 In captivity, primates are provisioned with food by human caretakers and so have reduced choice over

493 the options available to them and, unlike wild primates that spend a large proportion of their waking hours

494 foraging for food, captive primates are typically fed following a regular schedule (Bloomsmith \& Lambeth,

495 1995). Therefore, this study was not only of academic interest but also created novel foraging enrichment for

496 this group of chimpanzees and encouraged them to explore their environment to find the best possible foods.

497 Indeed, a recent review of tool-use innovation by wild primates suggested that chimpanzees are more likely to

498 innovate when the local ecology supports it (i.e. invention due to opportunity, not necessity, Koops, Visalberghi

499 \& van Schaik, 2014) and, in modest terms, we provided our zoo-housed group of chimpanzees with

500 opportunities for innovation and discovery. This study also highlighted the individual differences among the

501 chimpanzees such that some were quick to discover new options while others took longer to exchange tokens

502 at all, but with such a small sample, further studies are required to determine how generalizable our findings

503 are. These individual differences are likely a combination of the chimpanzees' rank (Reader \& Laland, 2001)

504 and their personality characteristics (Freeman \& Gosling, 2010), which have been shown to correlate with

505 chimpanzee problem-solving abilities in other foraging studies (Massen et al., 2013; Hopper et al., 2014b).

506

507 Acknowledgements

508 Thanks to Katherine Cronin for her constructive feedback on earlier drafts of this article and for the comments

509 given to us by three reviewers and the editor, Jennifer Vonk. Additionally, we thank Maureen Leahy, Michael

510 Brown-Palsgrove, and the animal care staff in the Regenstein Center for African Apes at Lincoln Park Zoo, for

511 making this research possible and for providing the highest level of care for the animals housed there. We are

512 also grateful to Marisa Shender for assisting with data collection and to numerous Fisher Center interns for 
513 collecting video footage during test sessions. We also thank Sara Skiba who blind coded footage for us and

514 Andrew Steets for his expert assistance with R and ggplot2.

515

516 References

517 Addessi E, Crescimbene L, Visalberghi E. 2007. Do capuchin monkeys (Cebus apella) use tokens as symbols?

518 Proceedings of the Royal Society of London: Biological Sciences. 274:2579-2585.

519 Addessi E, Mancini A, Crescimbene L, Visalberghi E. 2011. How social context, token value, and time course affect token exchange in capuchin monkeys (Cebus apella). International Journal of Primatology 32(1):83-98.

Avarguès-Weber A, Chittka L. 2014. Local enhancement or stimulus enhancement? Bumblebee social learning results in a specific pattern of flower preference. Animal Behaviour 97:185-191.

Ban SD, Boesch C, Janmaat KRL. 2014. Taï chimpanzees anticipate high-valued fruit trees from further distances. Animal Cognition 17(6):1353-1364.

Bautista LM, Tinbergen J, Kacelnik A. 2001. To walk or to fly? How birds choose among foraging modes. Proceedings of the National Academy of Sciences 98(3):1089-1094. problem. Animal Cognition. 15(2):239-250.

Bloomsmith MA, Lambeth SP. 1995. Effects of predictable versus unpredictable feeding schedules on chimpanzee behavior. Applied Animal Behaviour Sciences 44(1):65-74.

Boinski S, Timm RM. 1985. Predation by squirrel monkeys and double-toothed kites on tent-making bats.

534 Boesch C, Boesch H. 1984. Mental map in wild chimpanzees: an analysis of hammer transports for nut cracking. Primates 25(2):160-170. 
536 Bonnie KE, Horner V, Whiten A, de Waal FBM. 2007. Spread of arbitrary conventions among chimpanzees: a controlled experiment. Proceedings of the Royal Society of London: Biological Sciences 274(1608):367372.

539 540 behavior in a captive environment. American Journal of Primatology 74:661-668.

Brosnan SF, de Waal FBM. 2004. Socially learned preferences for differentially rewarded tokens in the brown capuchin monkey (Cebus apella). Journal of Comparative Psychology 118(2):133-139.

Calcutt SE, Lonsdorf EV, Bonnie KE, Milstein MS, Ross SR. 2014. Captive chimpanzees share diminishing resources. Behaviour 151(14):1967-1982.

Cronin KA, Pieper BA, van Leeuwen EJC, Mundry R, Haun DBM. 2014. Problem solving in the presence of others: how rank and relationship quality impact resource acquisition in chimpanzees (Pan troglodytes). PLoS ONE 9(4):e93204.

Dean LG, Kendal RL, Schapiro SJ, Thierry B, Laland KN. 2012. Identification of the social and cognitive processes underlying human cumulative culture. Science 335(6072):1114-1118.

Drea CM, Wallen K. 1999. Low-status monkeys "play dumb" when learning in mixed social groups. Proceedings of the National Academy of Sciences 96(22):12965-12960.

Evans TA, Beran MJ, Paglieri F, Addessi E. 2012. Delayed gratification for food and tokens in capuchin monkeys (Cebus apella) and chimpanzees (Pan troglodytes): when quantity is salient, symbolic stimuli do not improve performance. Animal Cognition 15:539-548.

Finestone E, Bonnie KE, Hopper LM, Vreeman VM, Lonsdorf EV, Ross SR. 2014. The interplay between individual, social, and environmental influences on chimpanzee food choices. Behavioural Processes 105:71-78. 
559 Freeman HD, Gosling SD. 2010. Personality in nonhuman primates: a review and evaluation of past research.

560

561

562

563

564

565

566

567

568

569

570

571

572

573

574

575

576

577

578

579

580

581

582 American Journal of Primatology 71:1-19.

Froehlich J, Chen M, Smith I, Potter F. 2006. Voting with your feet: an investigative study of the relationship between place visit behavior and preference. In Dourish P, Friday A, eds. UbiComp 2006: Ubiquitous Computing. Berlin Heidelberg: Springer (4206):333-350.

Hardus M, Lameira A, Wich S, de Vries H, Wahyudi R, Shumaker R, Menken SBJ. 2015. Effect of repeated exposures and sociality on novel food acceptance and consumption by orangutans. Primates 56(1):2127.

Hopper LM, Holmes AN, Williams LE, Brosnan SF. 2013. Dissecting the mechanisms of squirrel monkey (Saimiri boliviensis) social learning. PeerJ 1:e13.

Hopper LM, Lambeth SP, Schapiro SJ, Brosnan SF. 2014a. Social comparison mediates chimpanzees' responses to loss, not frustration. Animal Cognition 17(6):1303-1311.

Hopper LM, Price SA, Freeman HD, Lambeth SP, Schapiro SJ, Kendal RL. 2014b. Influence of personality, age, sex, and estrous state on chimpanzee problem-solving success. Animal Cognition 17(4):835-847.

Hopper LM, Schapiro SJ, Lambeth SP, Brosnan SF. 2011. Chimpanzees' socially maintained food preferences indicate both conservatism and conformity. Animal Behaviour 81:1195-1202.

Hopper LM, Tennie C, Ross SR, Lonsdorf EV. 2015. Chimpanzees create and modify probe tools functionally: a study with zoo-housed chimpanzees. American Journal of Primatology 77(2):162-170.

Horner V, Proctor D, Bonnie KE, Whiten A, de Waal FBM. 2010. Prestige affects cultural learning in chimpanzees. PLOS ONE 5(5):e10625.

Howard AM, Fragaszy DM. 2014. Multi-step routes of capuchin monkeys in a laser pointer traveling salesman task. American Journal of Primatology 76:828-841.

Hrubesch C, Preuschoft S, van Schaik CP. 2009. Skill mastery inhibits adoption of observed alternative solutions among chimpanzees (Pan troglodytes) Animal Cognition 12(2):209-216. 
583 Janmaat KRL, Ban SD, Boesch C. 2013. Chimpanzees use long-term spatial memory to monitor large fruit trees 584 and remember feeding experiences across seasons. Animal Behaviour 86(6):1183-1205.

585 Janmaat KRL, Byrne RW, Zuberbühler K. 2006. Primates take weather into account when searching for fruits. Current Biology 16:1232-1237.

Janmaat KRL, Polansky L, Ban SD, Boesch C. 2014. Wild chimpanzees plan their breakfast time, type, and

Janson C. 2007. Experimental evidence for route integration and strategic planning in wild capuchin monkeys. location. Proceedings of the National Academy of Sciences 111(46):16343-16348.

$$
\text { Animal Cognition 10(3):341-356. }
$$

Kendal RL, Hopper LM, Whiten A, Brosnan SF, Lambeth SP, Schapiro SJ \& Hoppitt W. 2015. Chimpanzees copy dominant and knowledgeable individuals: implications for cultural diversity. Evolution \& Human Behavior36:65-72.

Koops K, Visalberghi E, van Schaik CP. 2014. The ecology of primate material culture. Biology Letters 10: 20140508.

Kralik JD, Sampson WW. 2012. A fruit in hand is worth many more in the bush: steep spatial discounting by free-ranging rhesus macaques (Macaca mulatta). Behavioural Processes 89(3):197-202Lihoreau M, Chitkka L, Raine NE. 2011. Trade-off between travel distance and prioritization of high-reward sites in traplining bumblebees. Functional Ecology 25(6):1284-1292.

Lonsdorf EV, Ross SR, Linick SA, Milstein MS, Melber TN. 2009. An experimental, comparative investigation of tool use in chimpanzees and gorillas. Animal Behaviour 77:1119-1126.

Manrique HM, Völter CJ, Call J. 2013. Repeated innovation in great apes. Animal Behaviour 85:195-202.

Marshall HH, Carter AJ, Ashford A, Rowcliffe JM, Cowlishaw G. 2015. Social effects on foraging behavior and success depend on local environmental conditions. Ecology and Evolution 5(2):475-492. 
605 Massen JJM, Antonides A, Arnold A-MK, Bionda T, Koski SE. 2013. A behavioral view on chimpanzee

606

607

608

609

610

611

612

613

614

615 personality: exploration tendency, persistence, boldness, and tool-orientation measured with group experiments. American Journal of Primatology 75:947-958.

Menzel EW. 1973. Chimpanzee spatial memory organization. Science 182(4115):943-945.

Mikolasch S, Kotrschal K, Schloegl C. 2012. The influence of local enhancement on choice performances in African Grey parrots (Psittacus erithacus) and jackdaws (Corvus monedula). Journal of Comparative Psychology 126(4):399-406.

Perrings C, Hannon B. 2001. An introduction to spatial discounting. Journal of Regional Science 41(1):23-38.

Pianka ER. 1997. Animal foraging: past, present and future. Trends in Ecology and Evolution 12:360-364.

Plante S, Colchero F, Calmé S. 2014. Foraging strategy of a Neotropical primate: how intrinsic and extrinsic factors influence destination and residence time. Journal of Animal Ecology 83:116-125.

Pollet TV, Roberts SGB, Dunbar RIM. 2013. Going that extra mile: individuals travel further to maintain face-toface contact with highly related kin than with less related kin. PLoS ONE 8(1):e53929.

Pyke GH. 1984. Optimal foraging theory: a critical review. Annual Review of Ecology and Systematics 15:523575.

R Development Core Team (2010) R: A language and environment for statistical computing. Vienna, Austria: R Foundation for Statistical Computing. Retrieved from http://www.R-project.org

Rapaport LG, Brown GR. 2008. Social influences on foraging behavior in young nonhuman primates: Learning what, where, and how to eat. Evolutionary Anthropology 17(4):189-201.

Reader SM, Laland KN. 2001. Primate innovation: sex, age and social rank differences. International Journal of Primatology 22(5):787-805.

Reilly MP, Posadas-Sánchez D, Kettle LC, Killeen PR. 2012. Rats (Rattus norvegicus) and pigeons (Columbia livia) are sensitive to the distance to food, but only rats request more food when distance increases. Behavioural Processes 91:236-243. 
629 Romley JA, Goldman DP. 2011. How costly is hospital quality? A revealed-preference approach. The Journal of

630

631

633

636 Industrial Economics 59(4):578-608.

Stevens JR, Rosati AG, Ross KR, Hauser MD. 2005. Will travel for food: spatial discounting in two new world monkeys. Current Biology 15(20):1855-1860.

Takahashi K, Masuda R, Yamashita Y. 2013. What to copy: the key factor of observational learning in striped jack (Pseudocaranx dentex) juveniles. Animal Cognition 17(2):495-501.

van de Waal E, Borgeaud C, Whiten A. 2013. Potent social learning and conformity shape a wild primate's foraging decisions. Science 340(6131):483-485.

van de Waal E, Bshary R, Whiten A. 2014. Wild vervet monkey infants acquire the food-processing variants of their mothers. Animal Behaviour 90:41-45.

van Leeuwen EJC, Cronin KA, Schütte S, Call J, Haun DBM. 2013. Chimpanzees (Pan troglodytes) flexibly adjust their behaviour in order to maximize payoffs, not to conform to majorities. PLOS ONE 8(11):e80945.

Visalberghi E, Addessi E. 2000. Seeing group members eating a familiar food enhances the acceptance of novel foods in capuchin monkeys. Animal Behaviour 60(1):69-76.

Wagner KE, Ross SR. 2013. Female sexual state influences the cognitive test performance of zoo-living chimpanzees (Pan troglodytes) but not gorillas (Gorilla gorilla gorilla). American Journal of Primatology 75(S1):44.

Webster MM, Laland KN. 2013. Local enhancement via eavesdropping on courtship displays in male guppies, Poecilia reticulata. Animal Behaviour 86(1):75-83.

Whiten A, Horner V, de Waal FBM. 2005. Conformity to cultural norms of tool use in chimpanzees. Nature 437:737-740.

Wickham H. 2009. ggplot2: Elegant Graphics For Data Analysis. Springer New York. Yamamoto S, Humle T, Tanaka M 2013. Basis for cumulative cultural evolution in chimpanzees: social learning of a more efficient tool-use technique. PLoS ONE 8(1):e55768. 


\section{1}

The chimpanzees' indoor enclosure and the locations where they could exchange tokens for food rewards during the study.

A plan of the chimpanzees' indoor enclosure at the Regenstein Center for African Apes, Lincoln Park Zoo also showing part of the visitor floor. Location A was where the chimpanzees could collect the PVC tokens from one of two hoppers hung on their cage mesh and, in phases 1 and 3 , where they could exchange the tokens with a researcher to obtain lesspreferred carrot pieces (CLOSE location, Table 1). Location B, which was a 10m distance from location A following the shortest route, was where the chimpanzees could exchange tokens with the researchers for more-preferred grapes in phases 1 and 3 (FAR location) and lesspreferred carrot pieces in phase 2 (when it was the CLOSE location, Table 1). For footage of the chimpanzees carrying tokens from location A to location B in phase 3 , as viewed from the visitor floor, go to http://youtu.be/bl-byx754Al. Location C was where the chimpanzees could exchange tokens with the researchers for more-preferred grapes in phase 2 (FAR location, Table 1). To reach this location the chimpanzees had to walk from $A$ for a minimum of $9 \mathrm{~m}$ and then climb $6 \mathrm{~m}$ to reach a mesh panel at the mezzanine level (15m total); for footage of a chimpanzee traveling from location $\mathrm{A}$ to $\mathrm{C}$ while carrying tokens, go to: http://youtu.be/mC34z6vxXhk. Both locations A and B were at ground-level, while location C was $6 \mathrm{~m}$ above ground-level. Each exchange location was a discrete area, separated from other exchange locations by a minimum of $10 \mathrm{~m}$, and the size of the mesh panels through which the chimpanzees could exchange tokens at these locations was $1.7 \mathrm{~m} \times 2 \mathrm{~m}$ (location A), $1 \mathrm{~m} \times 2 \mathrm{~m}$ (location B) and $1 \mathrm{~m} \times 1 \mathrm{~m}$ (location C). 


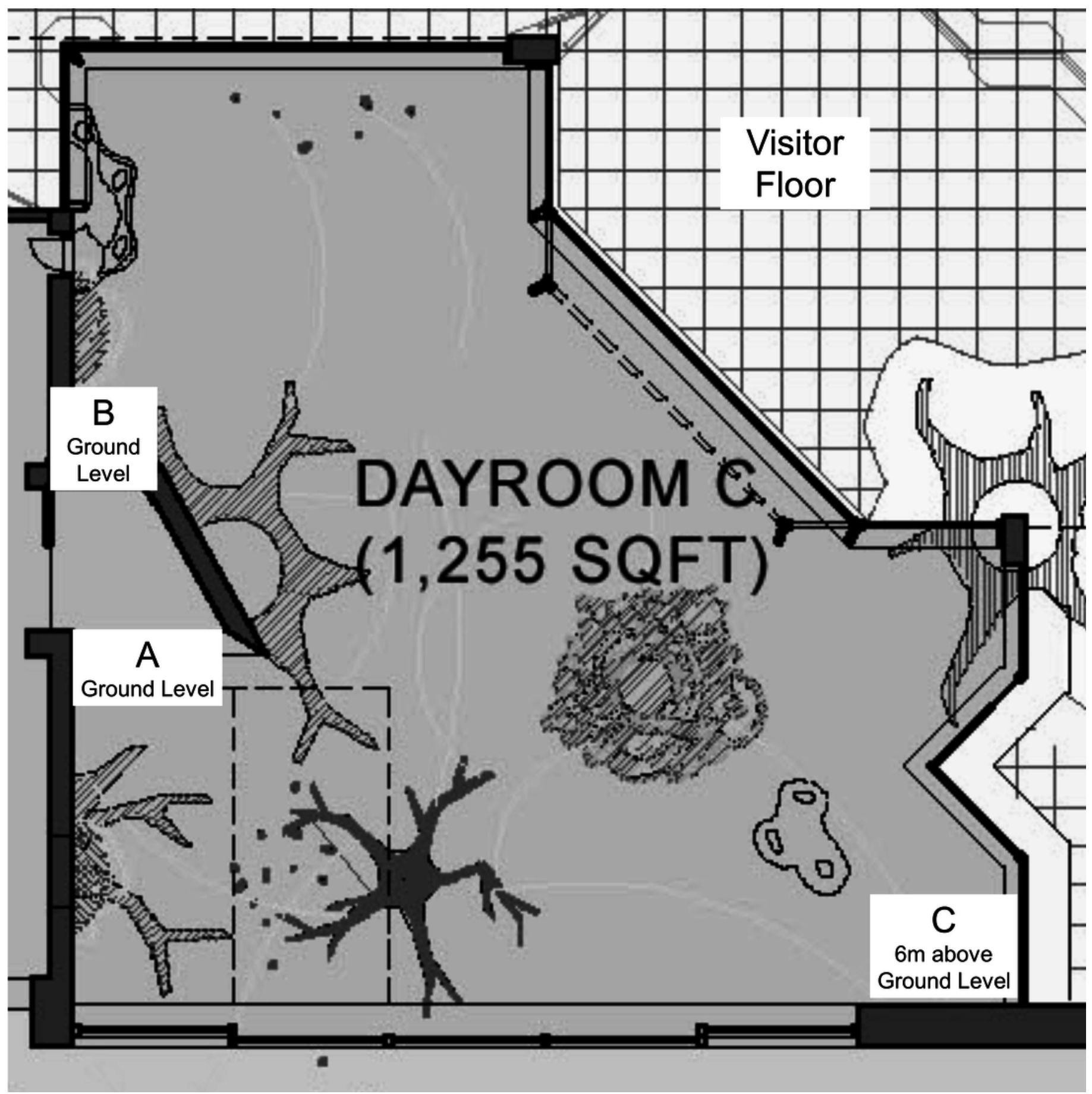




\section{2}

Cumulative total of token exchanges made by the chimpanzees at both the CLOSE (top) and FAR (bottom) exchange locations throughout the 90 sessions.

All six chimpanzees exchanged multiple tokens throughout this study although the alpha male $(\mathrm{HA})$ only exchanged tokens in phase 3 . Note too the plateau of exchanges in phase 2 (sessions 31-60) at the CLOSE location when a total of only 34 exchanges were made (by $\mathrm{CH}$ and OP, Table 2). During this phase, chimpanzees had to travel to obtain either reward, and so were required to carry their tokens $10 \mathrm{~m}$ to reach the CLOSE location, unlike in phase 1 (sessions 1-30) and phase 3 (sessions 61-90) in which no travel was required to reach the CLOSE location (chimpanzees could exchange their tokens where they collected them to obtain carrot pieces at location A)

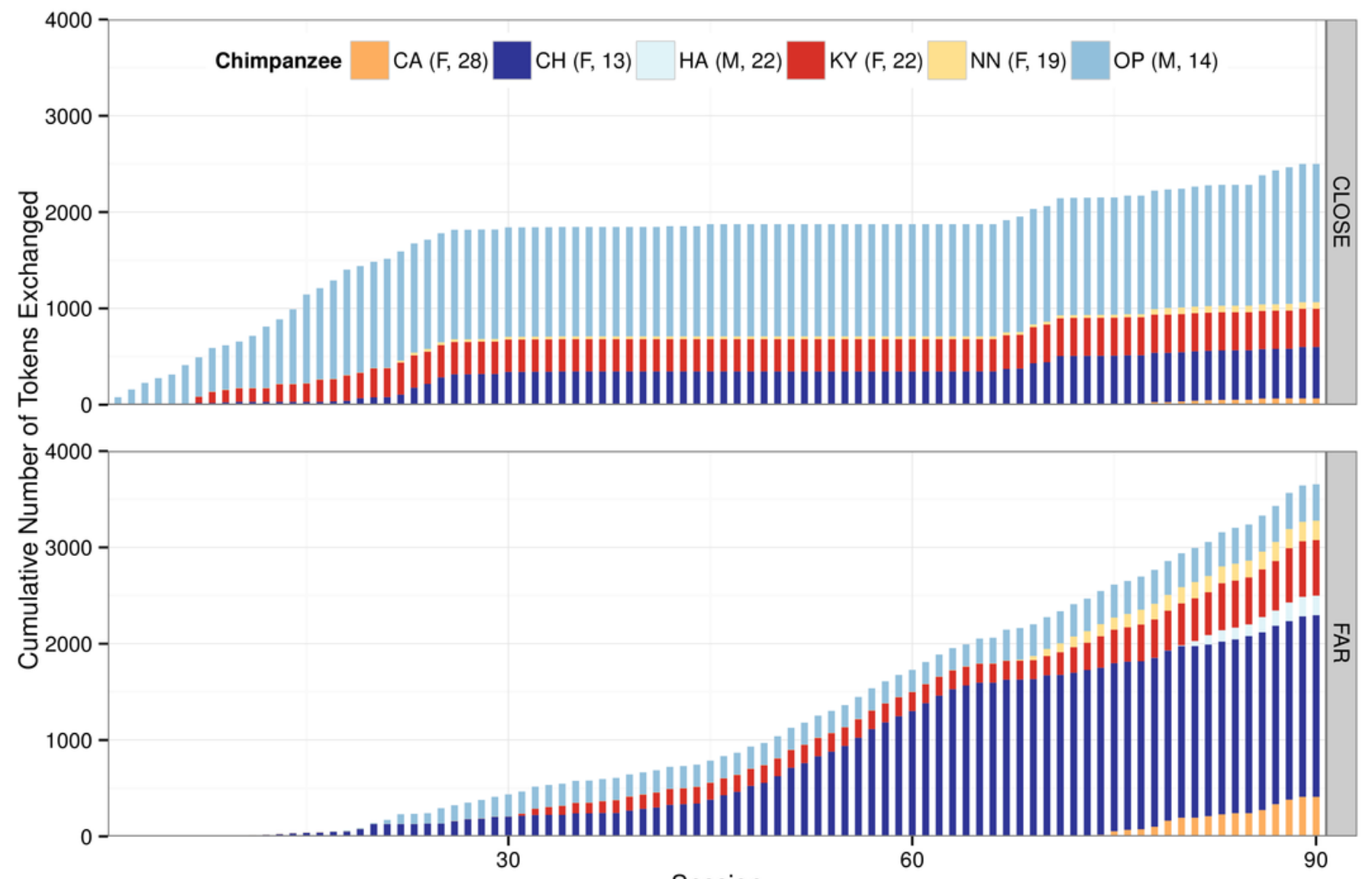




\section{3}

The total number of tokens that each of the chimpanzees exchanged at the CLOSE and FAR locations in phase 3

The ID code for each chimpanzee also provides information about their sex (M or F) and their age in years at the start of the study in January 2012. Note that male HA only exchanged tokens at the FAR location and never at the CLOSE location.

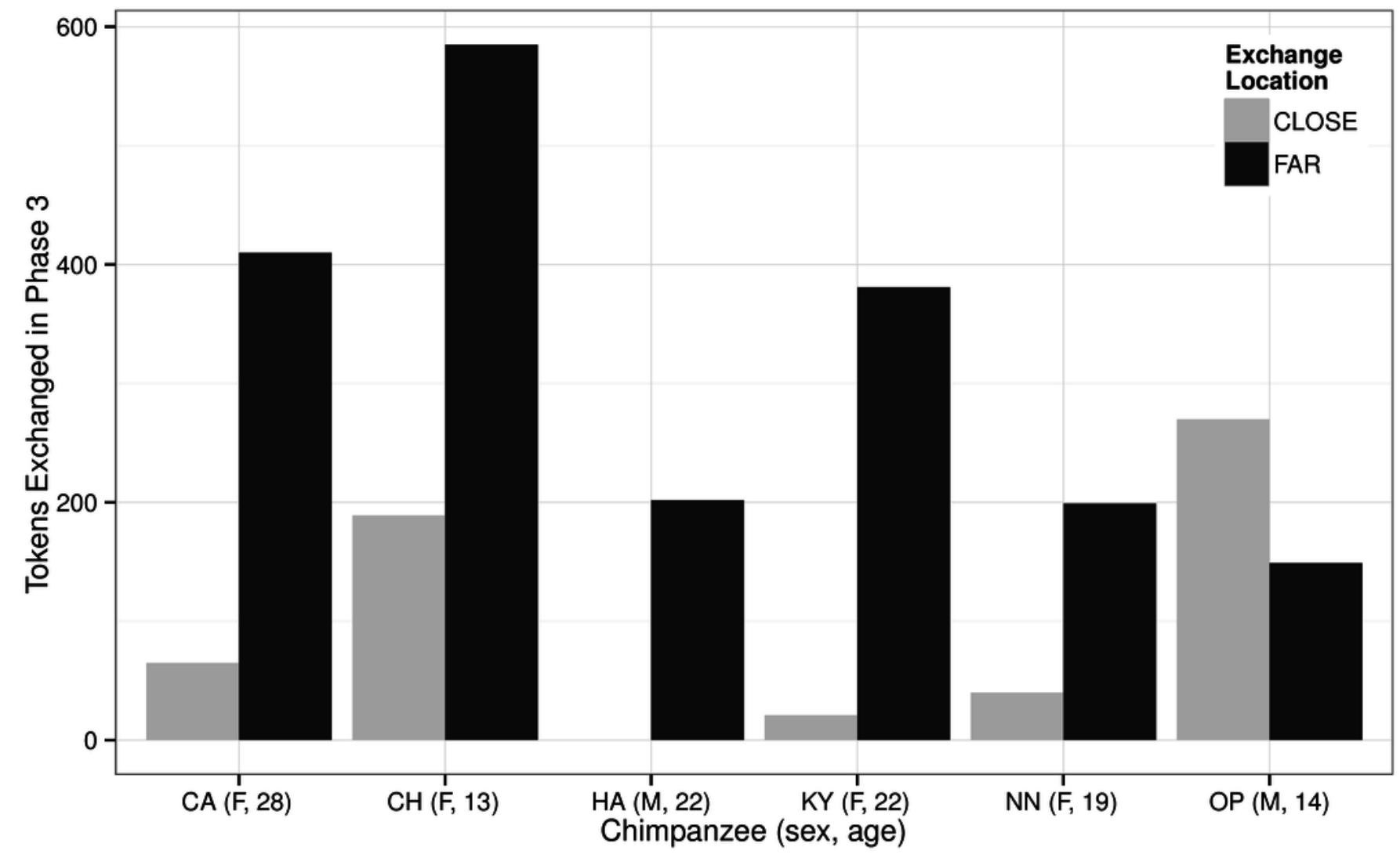


Table 1 (on next page)

An overview of the three experimental phases.

The food rewards that were available at each of the three locations (shown in Figure 1) in each of the three phases. Each phase was comprised of 30 30-minute sessions. The tokens, which the chimpanzees had to collect to exchange for the food rewards, were always available at location $A$. The distance from $A$ to $B$ was $\geq 10 \mathrm{~m}$ and the distance from $A$ to $C$ was $\geq 15 \mathrm{~m}$, including a $6 \mathrm{~m}$ climb to reach an elevated platform (Figure 1 ). 


\begin{tabular}{|l|l|l|l|}
\hline & Location A & Location B & Location C \\
\hline Phase 1 & Tokens + Carrot pieces & Grapes & \\
\hline Phase 2 & Tokens & Carrot pieces & Grapes \\
\hline Phase 3 & Tokens + Carrot pieces & Grapes & \\
\hline
\end{tabular}

2 


\section{Table 2 (on next page)}

The number of tokens exchanged by each of the six chimpanzees at each exchange location in each phase.

The ID code for each chimpanzee also provides information about their sex (M or F), their age in years at the start of the study in January 2012, and the number presented outside the brackets is their average rank score where $1=$ least dominant and $6=$ most dominant. 


\begin{tabular}{|l|r|r|r|r|r|r|}
\hline & \multicolumn{2}{|c|}{ Phase 1 } & \multicolumn{2}{c|}{ Phase 2 } & \multicolumn{2}{c|}{ Phase 3 } \\
\hline Chimpanzee & \multicolumn{1}{|c|}{ CLOSE } & \multicolumn{1}{|c|}{ FAR } & \multicolumn{1}{c|}{ CLOSE } & \multicolumn{1}{c|}{ FAR } & \multicolumn{1}{c|}{ CLOSE } & \multicolumn{1}{c|}{ FAR } \\
\hline CA $(F, 28) 4$ & 2 & 0 & 0 & 1 & 65 & 410 \\
\hline CH $(F, 13) 1$ & 340 & 206 & 4 & 1095 & 189 & 585 \\
\hline HA (M, 22) 6 & 0 & 0 & 0 & 0 & 0 & 202 \\
\hline KY (F, 22) 3 & 335 & 0 & 0 & 196 & 61 & 381 \\
\hline NN (F, 19) 4 & 29 & 2 & 0 & 1 & 40 & 199 \\
\hline OP (M, 14) 2 & 1135 & 227 & 30 & 0 & 270 & 149 \\
\hline
\end{tabular}

2 


\section{Table 3 (on next page)}

The number of exchanges that each subject observed at each location in each phase.

The first number is the total number of exchanges observed by a chimpanzee and the number shown in brackets is the number of exchanges observed by a chimpanzee before they made an exchange themselves at that location and ' - ' indicates that they never made an exchange at that location within that phase. The ID code for each chimpanzee also provides information about their sex ( $M$ or $F$ ), their age in years at the start of the study in January 2012, and the number presented outside the brackets is their average rank score where $1=$ least dominant and $6=$ most dominant. 


\begin{tabular}{|l|l|l|l|l|l|l|}
\hline & \multicolumn{2}{|c|}{ Phase 1 } & \multicolumn{2}{c|}{ Phase 2 } & \multicolumn{2}{c|}{ Phase 3 } \\
\hline Chimpanzee & \multicolumn{1}{|c|}{ CLOSE } & \multicolumn{1}{c|}{ FAR } & \multicolumn{1}{c|}{ CLOSE } & \multicolumn{1}{c|}{ FAR } & \multicolumn{1}{c|}{ CLOSE } & \multicolumn{1}{c|}{ FAR } \\
\hline CA $(F, 28) 4$ & $120(21)$ & $10(-)$ & $0(-)$ & $30(28)$ & $55(3)$ & $312(32)$ \\
\hline CH $(F, 13) 1$ & $256(49)$ & $7(0)$ & $0(0)$ & $158(0)$ & $43(0)$ & $314(0)$ \\
\hline HA $(\mathrm{M}, 22) 6$ & $20(-)$ & $19(-)$ & $2(-)$ & $9(-)$ & $6(-)$ & $237(125)$ \\
\hline KY (F, 22) 3 & $238(78)$ & $6(-)$ & $0(-)$ & $96(7)$ & $55(0)$ & $287(21)$ \\
\hline NN (F, 19) 4 & $317(19)$ & $9(0)$ & $0(-)$ & $157(69)$ & $32(7)$ & $482(0)$ \\
\hline OP (M, 14) 2 & $155(0)$ & $0(0)$ & $0(0)$ & $8(-)$ & $26(2)$ & $141(0)$ \\
\hline
\end{tabular}

